\title{
Síndrome de Lemierre, secundario a otitis media aguda. Revisión a partir de un caso
}

\section{Lemierre syndrome secondary to acute otitis media. Review from a case}

\author{
José T. Andrade D, José T. San Martín M², Carolina Grau L¹.
}

\begin{abstract}
RESUMEN
El síndrome de Lemierre (SL), una grave complicación, generalmente de las infecciones orofaríngeas. Se caracteriza por una inflamación agresiva del espacio parafaríngeo lateral, aparición de tromboflebitis de la vena yugular interna y el desarrollo de émbolos sépticos a distancia. Con un diagnóstico clínico y radiológico para su confirmación y posterior seguimiento, debe ser tratado con antibioterapia precoz y parenteral, además de debridación, drenaje quirúrgico de las posibles colecciones purulentas que presente y eventual anticoagulación.

En el siguiente trabajo, reportamos el caso de un paciente con esta rara, pero nuevamente emergente entidad, en donde se profundiza su estudio y se resume la literatura.
\end{abstract}

\begin{abstract}
Lemierre's syndrome (SL), generally, a serious complication of the oropharyngeal infections. Its characterized by an aggressive inflammation of the lateral parapharyngeal space, thrombophlebitis of the yugular internal vein and metastasic abscesses in different organs. With a clinical and radiological diagnosis for his confirmation and later followup, it must be treatedwith iv antibiotics and surgical drainage of the possible purulent collections that he presentsand eventual anticoagulation

In the following work, we bring the case of a patient with this rare, but again emergent entity, where his study is deepened and the literature is summarized.
\end{abstract}

\footnotetext{
${ }^{1}$ Residente Otorrinolaringología, Pontificia Universidad Católica de Chile.

2 Médico Cirujano.
} 


\section{INTRODUCCIÓN}

Considerada una enfermedad olvidada para algunos autores, por su relativa baja frecuencia de presentación en la era posantibiótica, el síndrome de Lemierre (SL), también denominado tromboflebitis séptica de la vena yugular interna, septicemia posanginal o necrobacilosis ${ }^{1}$, representa una entidad potencialmente fatal en ausencia de un pronto reconocimiento y adecuado manejo. La entidad se caracteriza por una agresiva inflamación del espacio parafaríngeo lateral, la aparición de tromboflebitis de la vena yugular interna y el desarrollo de émbolos sépticos a distancia. En la mayoría de los pacientes, como en las primeras descripciones, el proceso infeccioso inicial que conducirá a la posterior tromboflebitis séptica de la vena yugular interna, se localiza en orofaringe; sin embargo, se han reportado casos secundarios a otro tipo de infecciones de cabeza y cuello como mastoiditis, otitis media aguda y celulitis ${ }^{2,3}$. Si bien, en el $80 \%$ de los pacientes se ha identificado al Fusobacterium necrophorum como agente responsable, también puede haber otros microorganismos implicados y, en algunos casos, los cultivos pueden resultar negativos. Presentamos un caso de SL con el doble objetivo de recordar que la entidad no está erradicada, y siguen presentándose casos de la misma, y subrayar la importancia crucial que tiene un diagnóstico precoz para su curación.

\section{CASO CLÍNICO}

Paciente de sexo masculino, de 11 años de edad, sin antecedentes mórbidos. Presenta cuadro de Otitis Media Aguda (OMA) derecha, el cual no cede luego de completar 7 días de tratamiento efectivo, razón por la que consulta a servicio de urgencias. A la exploración el paciente presenta fiebre alta acompañada de dolor en región retroauricular e importante dificultad en la movilización cervical. Al examen físico la orofaringe y la nariz presentaban un aspecto normal. La otoscopia revelaba en el oído derecho tumefacción e inflamación del tímpano, con signos de ocupación de caja, y el oído izquierdo se encontraba normal. En el estudio de laboratorio inicial presenta Hcto: 30\% Hb: 9.8 Leucos: 12.100 Plaq: 433.000 PCR: 58 BUN: 6 Crea: 0.44 Albúmina: 2.8.

Se decide realizar TAC de cuello y cerebro (sin contraste), demostrándose lesión cavitada en espacio prevertebral derecho que impresionaba corresponder a adenopatías necrosadas sin compromiso de vía aérea, por lo que se manejó inicialmente con clindamicina y ceftriaxona endovenosos. Inicialmente evoluciona en buenas condiciones generales y afebril.

Posteriormente, se decide ampliar el estudio realizando nuevo TAC de cuello con contraste el que ahora mostraba trombosis de vena yugular común extensa, además de compromiso extenso de celdillas mastoideas a derecha (Figura 1) y, en los cortes que se alcanzaba a visualizar pulmón, múltiples nódulos pulmonares sólidos, algunos de ellos cavitados de distribución periférica, que impresionaban embolias sépticas (Figura 3). Se decide realizar eco cervical la cual confirma trombosis de vena yugular interna derecha y se diagnostica síndrome de Lemierre.

Con todo esto se amplió cobertura antibiótica, agregando ahora penicilina ev, con lo que el paciente evolucionó de manera favorable. Dado los focos pulmonares, se realizó radiografía de tórax que no evidenció alteraciones clínicamente relevantes, pero debido a la posibilidad de embolias sépticas a distancia, ya evidenciadas a nivel pulmonar. Se amplió estudio con cintigrama óseo para descartar osteomielitis, el cual finalmente no mostró focos y ecocardiograma que también descartó EBSA.

Además, por la trombosis extensa de vena yugular interna derecha (Figuras 2 y 4 ), con focos de embolia a distancia (pulmón), se decide iniciar anticoagulación con Clexane ${ }^{\circledR} 1 \mathrm{mg} / \mathrm{kg}$ el día.

Paciente completa en total 14 días de clindamicina yceftriaxona ev, además de 13 días de penicilina ev al momento del alta. Se decide completar tratamiento vía oral con amoxicilina/ac.clavulánico al menos 21 días y 4 semanas más con TACO. Se da de alta en buen estado general, afebril, asintomático y con examen pulmonar normal.

\section{DISCUSIÓN}

El síndrome de Lemierre, también conocido como septicemia posanginal o posamigdalina, se caracteriza por una inflamación agresiva del espacio parafaríngeo lateral, la aparición de tromboflebitis de la vena yugular interna y el desarrollo de émbolos sépticos a distancia ${ }^{4}$. 


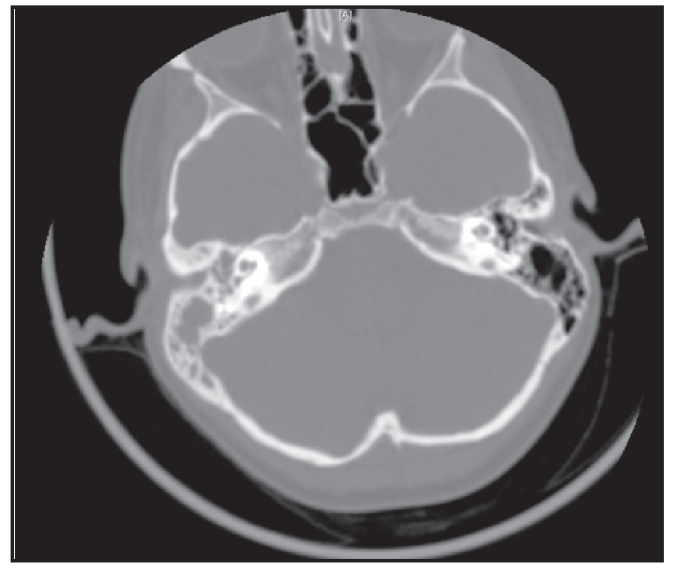

Figura 1. Celdillas mastoideas ocupadas por líquido.

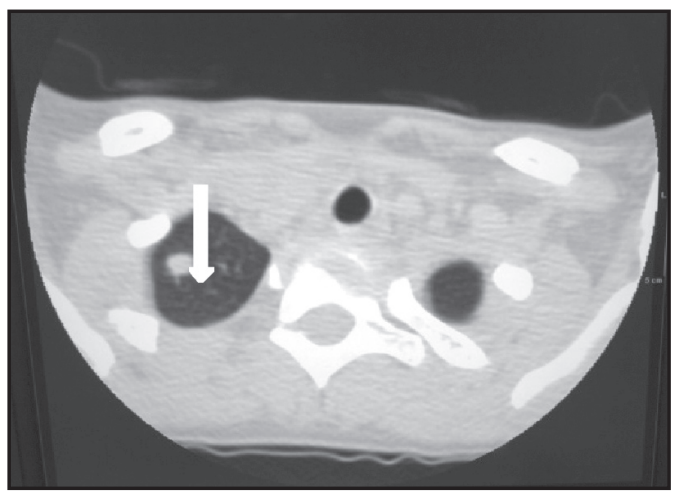

Figura 3. TAC de pulmón donde flecha indica foco de embolia séptica pulmonar.

Desde su descripción inicial antes de la era antibiótica en 1940, se reportaron más de 250 casos de bacteriemia por Fusobacterium necrophorum alrededor del mundo ${ }^{4}$. Sin embargo, luego del inicio del uso masivo de antibióticos por vía oral, la cantidad de casos reportados ha disminuido considerablemente. Entre los años 1950 y 1972, Bartlett y Finegold ${ }^{5}$, fueron incapaces de encontrar una sola publicación de algún caso de SL. Luego, en 1995 Leugers y Clover $^{6}$, reportan una serie de 40 casos de SL entre el año 1950 y 1995, ocurriendo los 2 últimos casos en las últimas dos décadas (1980s - 1990s). Todo esto nos muestra el panorama de una entidad que ha ido desapareciendo en las últimas décadas. Sin embargo, han ocurrido reportes en los que esta enfermedad presenta un leve aumento en su incidencia como el de Ramírez

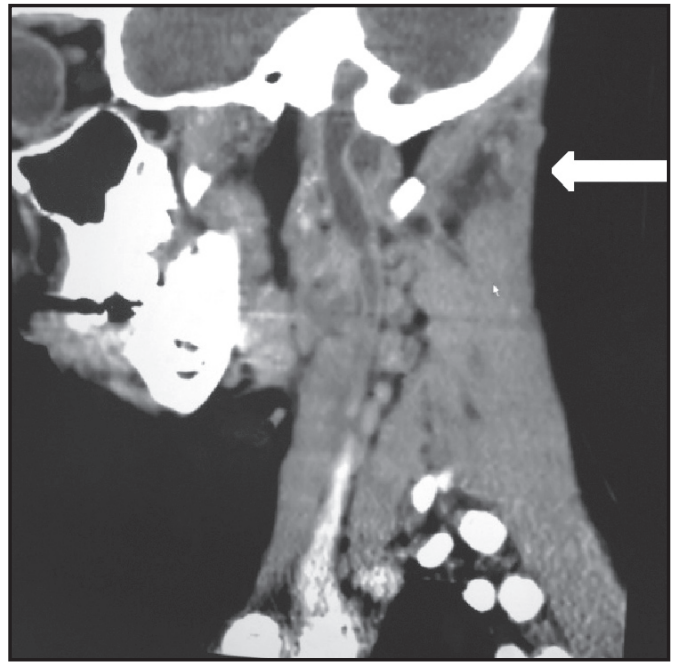

Figura 2. TAC con contraste. La flecha muestra la trombosis de la vena yugular interna.

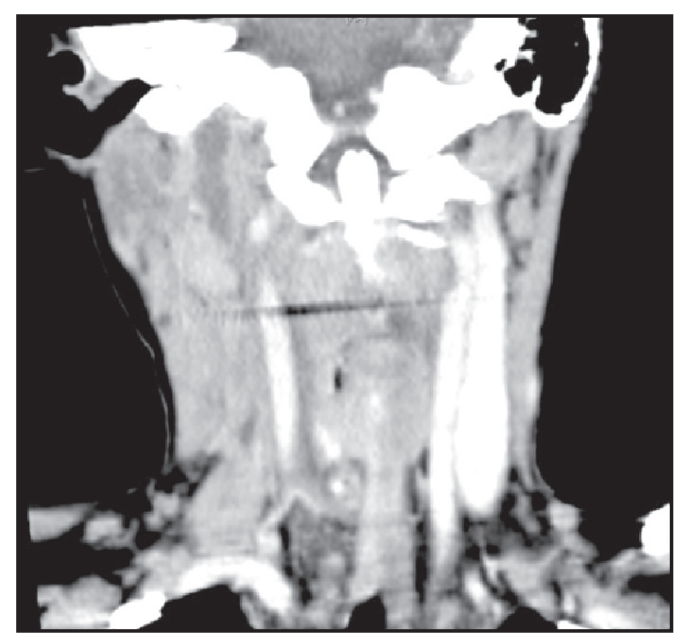

Figura 4. TAC de cuello con contraste, donde flecha indica la trombosis de vena yugular interna.

y cols ${ }^{7}$. Donde se evaluó la tasa de infecciones por bacteremia por $F$ necrophorum y los diagnósticos de SL en un hospital de niños en Wisconsin. Aquí se observó una incidencia de un aislamiento de este patógeno entre 1996 a 1999, el que aumento a 10 entre los años 2000 a 2002, con un diagnóstico de 5 casos formales de $\mathrm{SL}^{7}$. Este intrigante fenómeno se ha relacionado con los actuales protocolos restrictivos de uso de antibióticos en las infecciones faríngeas, la detección de cepas de Fusobacterium 
productoras de betalactamasa y por la tendencia creciente de los médicos generales a prescribir macrólidos (azitromicina o claritromicina), agentes sin acción anaerobicida, en el tratamiento de estas infecciones ${ }^{8}$. Por estas razones, algunos centros epidemiológicos tienen el convencimiento de que el SL es actualmente una entidad infradiagnosticada ${ }^{8}$.

En la inmensa mayoría de los cuadros con este síndrome, se trata de jóvenes con una infección faríngea que no remite pasados 3-7 días y que secomplica con la aparición de: fiebre alta, sepsis y aparición de signos de flogosis del espacio parafaríngeo lateral (EPL) con tromboflebitis de la VYI y, finalmente, focos sépticos a distancia9. Cuadro clínico clásico que se puede observar en otro caso presentado anteriormente por nuestra institución, el cual se presentó con un cuadro de odinofagia y fiebre, el cual progresó rápidamente a un absceso periamigdalino con trombosis de la vena yugular interna ipsilateral, embolias sépticas pulmonares y meningitis. Este se manejó con drenaje del absceso por punción, terapia antibiótica de amplio espectro y anticoagulación respondiendo favorablemente y siendo dado de alta en buenas condiciones luego de 35 días de hospitalización ${ }^{18}$.

Aunque algunos autores requieren la existencia previa de un cuadro amigdalar grave (angina de Plaut-Vincent), muchos de los casos se han descrito después de episodios subclínicos de infección orofaríngea aguda, ocurridos varios días antes de la diseminación séptica hematógena ${ }^{10}$. Además, se han descrito alguno casos de este síndrome como complicación secundaria a infecciones en otras regiones de cabeza y cuello, dentro de ellas la otitis media $^{11}$, como se pudo observar en nuestro paciente, alertando aún más al clínico en su sospecha ante pacientes con este tipo de síntomas y signos.

No se conocen los factores que hacen que el germen pase de producir una inflamación banal a aumentar su invasividad y penetrar retrógradamente en el EPL. El germen, una vez atravesada la mucosa, produciría intensa inflamación del EPL e invadiría Ios linfáticos de la zona (linfadenitis), los cuales están en íntimo contacto con la pared de las venas peritonsilares y VYI, que se verían afectadas sufriendo un proceso de tromboflebitis con la consiguiente embolización de material infectado ${ }^{9}$. Sin embargo, la tromboflebitis puede ocurrir en una variedad de vasos de la cabeza y cuello, incluyendo el plexo venoso parafaríngeo y las venas peritonsilares ${ }^{12}$. Este síndrome también se ha asociado a trombosis del seno cavernoso y trombosis del seno lateral ${ }^{12}$.

En virtualmente todos los casos de SL (90\%), cuando se presenta la tromboflebitis de la vena yugular, suelen ocurrir émbolos sépticos a distancia, siendo los pulmones el lugar más afectado. Otros sitios de embolización en orden decreciente de frecuencia son: articulaciones mayores, huesos, músculo, hígado, bazo y cerebro ${ }^{3}$.

El diagnóstico del SL es básicamente clínico, con alto grado de sospecha y apoyo en la imagenología como la ecografía doppler color y el TAC cervical con medio contraste. Estas son muy útiles para documentar las anomalías anatómicas subyacentes, como el edema o absceso del EPL y la trombosis de la VYI, pero se considera al TAC cervical, la prueba de imagen de elección dado a que presenta mayor sensibilidad que los ultrasonidos, tanto como para detectar el edema del EPL y la eventual trombosis de la $\mathrm{VYI}{ }^{13}$.

El tratamiento del SL se basa en la administración de antibióticos por vía venosa durante los primeros 7-10 días, seguidos por un periodo de terapia oral típicamente prolongado (3-6 semanas). El Fusobacterium es clásicamente sensible a penicilina $\mathrm{G}$ así como a otros antibióticos con actividad anaerobicida como: metronidazol, clindamicina e imipenem. La susceptibilidad a cefalosporinas, eritromicina y tetraciclinas es variable y es resistente al aztreonam y al trimetopin-sulafametoxazol, como también a los aminoglicósidos ${ }^{9,15}$. Sin embargo, fallas en el tratamiento con penicilina han sido reportadas por la producción de betalactamasas por parte de este microorganismo ${ }^{14,15}$. Es por esta razón, que varios autores y expertos recomiendan el uso de antibióticos con actividad antibetalactamasa y antianaeróbica, como lo son ampicilina-sulbactam, metronidazol y clindamicina $^{14,15}$. El otro pilar fundamental del tratamiento es el drenaje/desbridamiento quirúrgico cuando se detecta abscesificación de los focos metastásicos (artritis purulenta, empiema, etc.) ${ }^{3}$.

Por otra parte, la anticoagulación es un asunto controvertido y aún no zanjado. Existe el riesgo, al menos teórico, de que la misma terapia pueda facilitar la diseminación de la infección. Por otro lado, en algunos casos se ha observado una resolución más rápida del cuadro coincidiendo con el inicio de la anticoagulación con heparina ${ }^{16,17}$. Razón por la cual, 
se decidió iniciar esta terapia en nuestro paciente, al igual que un paciente reportado anteriormente en nuestro Hospital, en donde obtuvimos muy buenos resultados al iniciar esta terapia ${ }^{18}$. Sin embargo, aún no se realizan estudios controlados en donde se evalúe la real efectividad de esta terapia, por lo que se nos hace difícil realizar una recomendación formal con respecto a este tema.

\section{CONCLUSIONES}

Con la presentación de este caso, se exhibe un caso de síndrome de Lemierre secundario a otitis media, una causa poco frecuente de este mismo, pero que subraya la importancia de la sospecha clínica ante pacientes como el presentado, recalcando además, la importancia en la instauración del tratamiento oportuno para esta entidad potencialmente fatal.

\section{BIBLIOGRAFÍA}

1. García F. Infección orofaríngea y sépsis: ¿una relación olvidada? Emergencias 2006; 18: 228-30.

2. Chirinos Ja, Garcia J, Alcaide ML, Toledo G, Baracco GJ, LichtSteIn DM. Septic thrombophlebitis: diagnosis and management. Am J Cardiovasc Drugs 2006; 6: 9-14.

3. Chirinos Ja, Lichtstein D, Garcia J, Tamariz LJ. The evolution of Lemierre syndrome: report of 2 cases and review of the literature. Medicine 2002; 81: 458-65.

4. Lemierre A. On certain septicemias due to anaerobic organisms. Lancet 1936; 1: 701-3.

5. Bartlett JG, Finegold SM. Anaerobic pleuropulmonary infections. Medicine (Baltimore) 1972; 51: 413-50.

6. Leugers CM, Clover R. Lemierre syndrome: postanginal sepsis. J Am Board Fam Pract 1995; 8: 384-91.

7. Ramírez S, Hild TG, Rudolph CN, Sty JR, Kehl SC, Havens P, et al. Increased diagnosis of
Lemierre Syndrome and other Fusobacterium necrophorum infections at a children's Hospital. Pediatrics 2003; 112: 380-5.

8. Brazier JS, May V, Yusuf e, Dueren Bi. Fusobacterium necrophorum infections in England and Wales 1990-2000. J Med Microbiol 2002; 51: 269-72.

9. Sinave CP, Hardy GJ, Fardy PW. The Lemierre syndrome: Thrombophlebitis of the internal jugular vein secondary to oropharyngeal infection. Medicine 1989; 68: 85-94.

10. Fernández Jl, Dones JJ, Carvajal I, Carcelén M, Gutiérrez JM, Lea MC, et al. Síndrome de Lemièrre: descripción de dos nuevos casos y revisión de la literatura. An Med Interna 2002; 19 (3): 139-42.

11. Lina Rosique López, Francisco Rodríguez Domínguez, Pilar Navarro Paule y Alejandro Soler Valcárcel. Síndrome de Lemierre. Caso poco habitual. Acta Otorrinolaringol Esp 2007; 58(5): 217-8.

12. Stokroos RJ, Manni JJ, de KrulJk JR, SoudiJn ER. Lemierre syndrome and acute mastoiditis. Arch Otolaryngol Head Neck Surg 1999; 125: 589-91.

13. Cook RJ, Ashton RW, Aughenbaugh GL, Ryu JH. Septic pulmonary embolism: Presenting features and clinical course of 14 patients. Chest 2005; 128: 162-6.

14. Brook I. Infections caused by beta-lactamase producing Fusobacterium spp in children. Pediatr Infect Dis J 1993; 12: 532-3.

15. Golpe R, Marin B, Alonso M. Lemierre's syndrome (necrobacillosis). Postgrad Med J 1999; 75: 141-4.

16. Merlo González Ve, Martín Parra C, Marina Martínez L, Ayensa Rincón A, Moreno Otero Ja, AgUILAR Florit J. Tromboflebitis séptica de la vena yugular interna secundaria a patología orofaríngea. Med Intensiva 1998; 22: 78-80.

17. Nadkarni MD, Verchick J, O'Neill JC. Lemierre syndrome. J Emerg Med 2005; 28: 297-9.

18. Matías Winter D, Andrea Saavedra B, Carolina Grau L, Jorge Caro L. Síndrome de Lemierre, reporte de un caso. Rev Otorrinolaringol Cir Cabeza Cuello 2013; 73: 164-8.

\footnotetext{
Dirección: Dr. José Tomás Andrade D.

Servicio Otorrinolaringología, Pontificia Universidad Católica de Chile

Santiago - Chile - Fono: 96097271

E mail: tomasandraded@gmail.com
} 